Available online at GSC Online Press Directory

GSC Biological and Pharmaceutical Sciences

e-ISSN: 2581-3250, CODEN (USA): GBPSC2

(RESEARCH ARTICLE)

\title{
In vitro influence of metronidazole on the activities of ciprofloxacin against clinically important bacterial isolates
}

\author{
Adeoye-Isijola Morenike Olutumbi, Osopale Babasola Adewunmi and Olajuyigbe Olufunmiso Olusola * \\ Department of Microbiology, School of Science and Technology, Babcock University, PMB 4005, Ilisan-Remo, Ogun State, \\ Nigeria.
}

Publication history: Received on 15 January 2019; revised on 01 March 2019; accepted on 04 March 2019

Article DOI: https://doi.org/10.30574/gscbps.2019.6.3.0006

\begin{abstract}
A continued in vitro investigation into drug-drug interaction is necessary to avert dangerous drug combinations and achieve effective therapy at lower drug concentrations. In this study, the interactions of ciprofloxacin and metronidazole and the effectiveness of the antibacterial combinations against selected bacterial strains were investigated by agar diffusion, macrobroth dilution and the checkerboard assay methods. The results showed that the combined antibiotics had better antibacterial effects than those produced by the ciprofloxacin while no inhibition zone was produced by metronidazole at the concentrations used. At the highest concentration of the combination, the inhibition zones ranged between $28 \pm 1.00 \mathrm{~mm}$ and $37 \pm 1.00 \mathrm{~mm}$ while Plesiomonas shigelloidis was highly resistant. While the minimum inhibitory concentrations (MICs) of ciprofloxacin ranged between 0.05 and $3.13 \mu \mathrm{g} / \mathrm{ml}$ and those of metronidazole were between 15.63 and $62.5 \mu \mathrm{g} / \mathrm{ml}$, the MICs for the antibacterial combinations were between 0.02/0.12 and 3.13/1.56 (Cip/Met) $\mu \mathrm{g} / \mathrm{ml}$ against all the test microorganisms. The percentage reduction in the MICs showed that while the MICs of ciprofloxacin were reduced by between 0 and $96.87 \%$, those of metronidazole were reduced by between 0 and 99.80 $\%$ when the two antibiotics were combined. The interaction of ciprofloxacin and metronidazole was more of synergy $(55.56 \%)$ than additive/indifference (44.4\%). The FICI indicating synergistic interaction ranged between 0.035 and 0.375 , the FICI indicating additive/indifference interactions ranged between 0.532 and 1.25 . No antagonistic interaction was recorded. The lack of antagonism between these antibacterial agents suggested that their combination would be an improved therapy over the use of each antibiotic individually and may be useful in attaining better therapeutic effects in infections where polymicrobial and resistant bacterial strains may be involved.
\end{abstract}

Keywords: Antibiotics; Bacterial infections; Drug-drug interactions; Synergism; Additive interaction

\section{Introduction}

Before the early $20^{\text {th }}$ century, treatments of infections were based primarily on medical folklore. Mixtures with antimicrobial properties that were used to treat infections included selected mold and plant materials and their extracts. Conversely, the discovery of antimicrobial drugs in the 1960s [1] and industrial synthesis of antimicrobial agents began as a science that rewrote the story of antibiotic chemotherapy. Today, antibiotics, chemotherapeutic agents occurring as a by-product of the metabolic activity of bacteria or fungi [2], are used to kill or prevent the growth of bacteria without destroying the cells in the body. While the global escalation in both community- and hospital-acquired antimicrobialresistant bacteria is threatening the ability to effectively treat patients and highlighting the need for continued surveillance, more appropriate antimicrobial prescribing, prudent infection control and new treatment alternatives [35], available therapeutic options for antibiotic-resistant organisms are severely limited as these organisms frequently display a multidrug-resistant phenotype [4-6]. The emergence of these multidrug-resistant bacteria, however, has posed threat worldwide to infection therapy [7].

\footnotetext{
${ }^{*}$ Corresponding author

E-mail address: funmijuyigbe12@yahoo.com
} 
Although antibiotic resistance has developed among some bacterial strains during treatment with these agents [8], the prescription and use of multiple drugs to deal with concomitant multiple diseases have become a norm [9,10]. Chait et al. [11], Hegreness et al. [12] and Michel et al. [13] indicated that antagonistic drug pairs can slow down and possibly, even, reverse the evolution of drug resistance. Phillips et al. [14] and Mani et al. [15] reported that interactions resulting from combining these drugs have been classified as synergistic, additive or antagonistic depending on whether the combined effect of two drugs on bacterial growth is greater than, equal to or less than predicted on the basis of the individual effects. Thus, combination therapies are most often given empirically, in the clinics, for synergy amplifications and diminishment of possible adverse effects, to arrest the progression of infections prior to getting results of laboratory investigations and to provide broad-spectrum coverage until the causative pathogens are isolated and identified [16]. However, as the number of medications taken by the individual patient increases and more and more patients are receiving multiple drug therapies for acute and chronic conditions or diseases, so does the potential for drug-drug interactions having clinically important consequences especially in elderly patients which are susceptible by virtue of polypharmacy, comorbid illness and treatment by multiple physicians $[17,18]$, changes in pharmacokinetics and pharmacodynamic parameters [19,20] and impairment of organ functions [21].

Consequential to increasing concern for bacterial resistance, drug interactions and adverse drug reactions which may include cognitive impairment, hospitalization and institutionalization, mortality and rising healthcare costs [22, 23], the pharmaceutical industry and regulatory agencies have indicated the need to conduct in vitro and in vivo drug interaction studies as a form of pharmacovigilance. Hence, considering the rate of introduction of new drugs and the burgeoning appreciation of the importance of pharmacogenetics [24, 25], it is unrealistic to expect clinicians to memorize the thousands of drug-drug interactions and their significance while a continued in vitro investigation into drug-drug interaction is necessary to avert dangerous drug combinations as well as achieving effective therapy at lower drug concentrations. This study, therefore, aimed at investigating the interactions of ciprofloxacin and metronidazole and the effectiveness of the antibacterial combinations against some selected bacterial strains.

\section{Material and methods}

\subsection{Test microorganisms}

The eighteen American Type Culture Collection (ATCC) pure cultures of bacteria used for this study were obtained from the Department of Microbiology and Biochemistry, University of Fort Hare, South Africa. They include Micrococcus luteus, Bacillus subtilis KZN, Shigella flexneri KZN, Enterococcus faecalis KZN, Enterococcus faecalis 29212, Proteus vulgaris ATCC 6830, Escherichia coli ATCC 8739, Shigella sonnei ATCC 29930, Serratia mercescens ATCC 9986, Enterobacter cloacae ATCC 13047, Klebsiella pneumoniae ATCC 4352, Klebsiella pneumoniae ATCC 10031, Staphylococcus aureus NCT 6571, Aeromonas hydrophila ATCC 35654, Pseudomonas aeruginosa ATCC 15442, Plesiomonas shigelloides ATCC 51903, Pseudomonas aeruginosa ATCC 19582 and Acinetobacter calcaoceuticus anitratus UP.

\subsection{Antibiotics used}

Antibiotic powders of ciprofloxacin (Duchefa) and metronidazole (Duchefa) were used. Stock antibiotic solutions were prepared and dilutions made according to the CLSI (Clinical Laboratory Standardization Institute) method and manufacturer's recommendations [26].

\subsection{Antibiotic susceptibility testing-agar diffusion method}

Each of the isolates was standardized using colony suspension method. Each strain's suspension was matched with 0.5 McFarland standards to give a resultant concentration of $1.5 \times 10^{7} \mathrm{cfu} / \mathrm{ml}$. The antibiotic susceptibility testing was determined using the modified Kirby-Bauer diffusion technique [27] by swabbing the Mueller-Hinton agar (MHA) (Oxoids U.K) plates with the resultant saline suspension of each strain. Wells were then bored into the agar medium with heat sterilized $6 \mathrm{~mm}$ cork borer. The wells were filled with $100 \mu \mathrm{l}$ of (12.5 and $25 \mu \mathrm{g} / \mathrm{ml}$ ) of ciprofloxacin, (31.25 and $62.5 \mu \mathrm{g} / \mathrm{ml})$ of metronidazole and $(12.5+62.5$ and $25+125 \mu \mathrm{g} / \mathrm{ml})$ of the antibacterial combinations taking care not to allow spillage of the solutions onto the agar surface. The plates were allowed to stand for at least $1 \mathrm{~h}$ before being incubated at $37^{\circ} \mathrm{C}$ for $24 \mathrm{~h}$. The determinations were done in triplicates. After $24 \mathrm{~h}$ of incubation, the plates were examined for the presence of inhibition zones. The diameter of the inhibition zones produced by the antibiotics alone and their combinations were measured and interpreted using the CLSI zone diameter interpretative standards [28]. Synergism was considered when combinations exhibited inhibition zones increment of 0.5 mm above those produced by the individual antibiotics. 


\subsection{Determination of minimal inhibitory concentration (MIC)}

The minimum inhibitory concentrations (MICs) for the two antibiotics were determined in duplicate by the macrobroth dilution method in Mueller Hinton broth according to Clinical Laboratory Standardization Institute (CLSI) [26]. To determine the MICs of each antibiotic, different concentrations of ciprofloxacin $(0.05-50) \mu \mathrm{g} / \mathrm{ml}$ and metronidazole $(0.5-250) \mu \mathrm{g} / \mathrm{ml}$ were prepared by serial dilution in double strength Mueller Hinton broth. These concentrations were chosen on the basis that the maximum serum concentration of ciprofloxacin and metronidazole was reported to range between $1.2 \mu \mathrm{g} / \mathrm{ml}$ to $5.4 \mu \mathrm{g} / \mathrm{ml}$ [29]. To determine the effects of combining these antibiotics, $100 \mu \mathrm{g} / \mathrm{ml}$ of ciprofloxacin was combined with $500 \mu \mathrm{g} / \mathrm{ml}$ of metronidazole before being serially diluted in double strength Mueller Hinton broth to determine their MICs in combination. After the serial dilution, the broth solutions were inoculated with $100 \mu \mathrm{l}$ of each of the bacterial strains and incubated at $37^{\circ} \mathrm{C}$ for $24 \mathrm{~h}$. Blank Mueller Hinton broth was used as negative control. Each combination assay was performed two times. The MIC was defined as the lowest dilution that showed no growth in the Mueller Hinton broth.

\subsection{Determination of minimum bactericidal concentrations (MBC)}

The minimum bactericidal concentration (MBC) is identified by determining the lowest concentration of anti-bacterial agent that reduces the viability of the initial bacterial inoculum by $\geq 99.9 \%$. The MBC assays were carried out as described by Cheesbrough [30]. Here, antibiotic-free nutrient agar plates were inoculated with one loopful of culture taken from each of the first three broth cultures that showed no growth and the first growth-containing tube in the MIC tubes. The MBC plates were incubated at $37^{\circ} \mathrm{C}$ for $24 \mathrm{~h}$. After the incubation periods, the lowest concentrations of ciprofloxacin, metronidazole and their combinations that did not produce bacterial growth on the solid medium were regarded as their MBC values. This observation was matched with the MIC test tube that did not show evidence of growth after $48 \mathrm{~h}$ of incubation.

\subsection{Checkerboard assay}

The interactions between the two antibiotics were determined using the checkerboard as previously described [31]. The range of drug concentration used in the checkerboard assay was such that the dilution range encompassed the MIC for each drug used in the analysis. The fractional inhibitory concentration (FIC) was derived from the lowest concentrations of the two antibiotics in combination permitting no visible growth of the test organisms in the Mueller Hinton broth after an incubation for $24 \mathrm{~h}$ at $37^{\circ} \mathrm{C}$ [32]. FIC indices were calculated using the formula FIC index $=$ (MIC of ciprofloxacin in combination/MIC of ciprofloxacin alone) + (MIC of metronidazole in combination/MIC of metronidazole alone). In this study, synergy was defined as $\sum$ FIC $\leq 0.5$, additivity as $0.5<\sum$ FIC $\leq 1$, indifference as $1<$ $\sum$ FIC $\leq 4$ and antagonism as $\sum$ FIC $>4$ by the checkerboard method according to Petersen et al. [31].

\subsection{Statistical analysis}

All the data were subjected to one way analysis of variance (ANOVA) and the mean values were separated at $p<0.05$ using Duncan's Multiple Range Test. The one way ANOVA test was used to determine if there was any statistically significant difference in the size of inhibition zones for each bacterial isolate exposed to each antibiotic alone and the antibiotics combination. All statistical analyses were performed using SPSS software (2009).

\section{Results}

The antibacterial activities showed that some of the inhibition zones produced by the combination were wider than those produced by ciprofloxacin while metronidazole did not produce any inhibition zone at the concentrations used. Lack of inhibition zones indicated that the organisms were all resistant to metronidazole while Plesiomonas shigelloides ATCC 51903 was resistant to both metronidazole and ciprofloxacin. The combination of the two antibiotics at a concentration of $25+125 \mu \mathrm{g} / \mathrm{ml}$ gave the widest zones of inhibition of $37 \pm 1.00 \mathrm{~mm}$ and $36 \pm 1.00 \mathrm{~mm}$ for Micrococcus luteus and Serratia mercescens ATCC 9986 respectively. An average zone of inhibition of $34 \pm 1.00 \mathrm{~mm}$ was recorded for Staphylococcus aureus ATCC 6538, Klebsiella pneumoniae ATCC 4352, Shigella sonnei ATCC 29930, Shigella flexneri KZN and Acinetobacter calcaoceuticus UP. While an average inhibition zone of $31 \pm 1.00 \mathrm{~mm}$ was recorded for Aeromonas hydrophila ATCC 35654, Pseudomonas aeruginosa ATCC 19582, Enterococcus faecalis KZN, Enterobacter cloacae ATCC 13047 and Proteus vulgaris ATCC 6830, Plesiomonas shigelloides ATCC 51903 was resistant to the two antibiotics and their combinations (Table 1). 


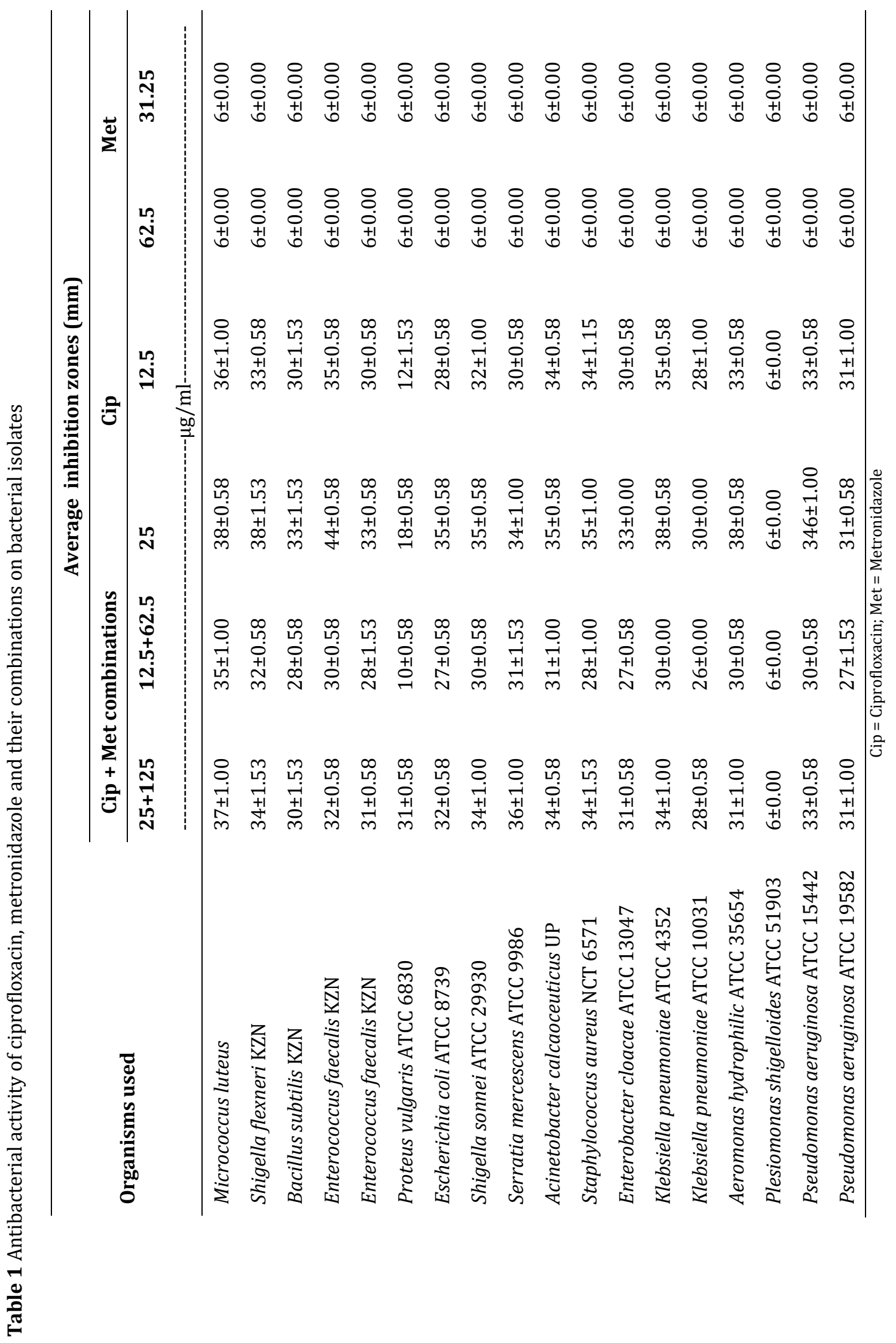




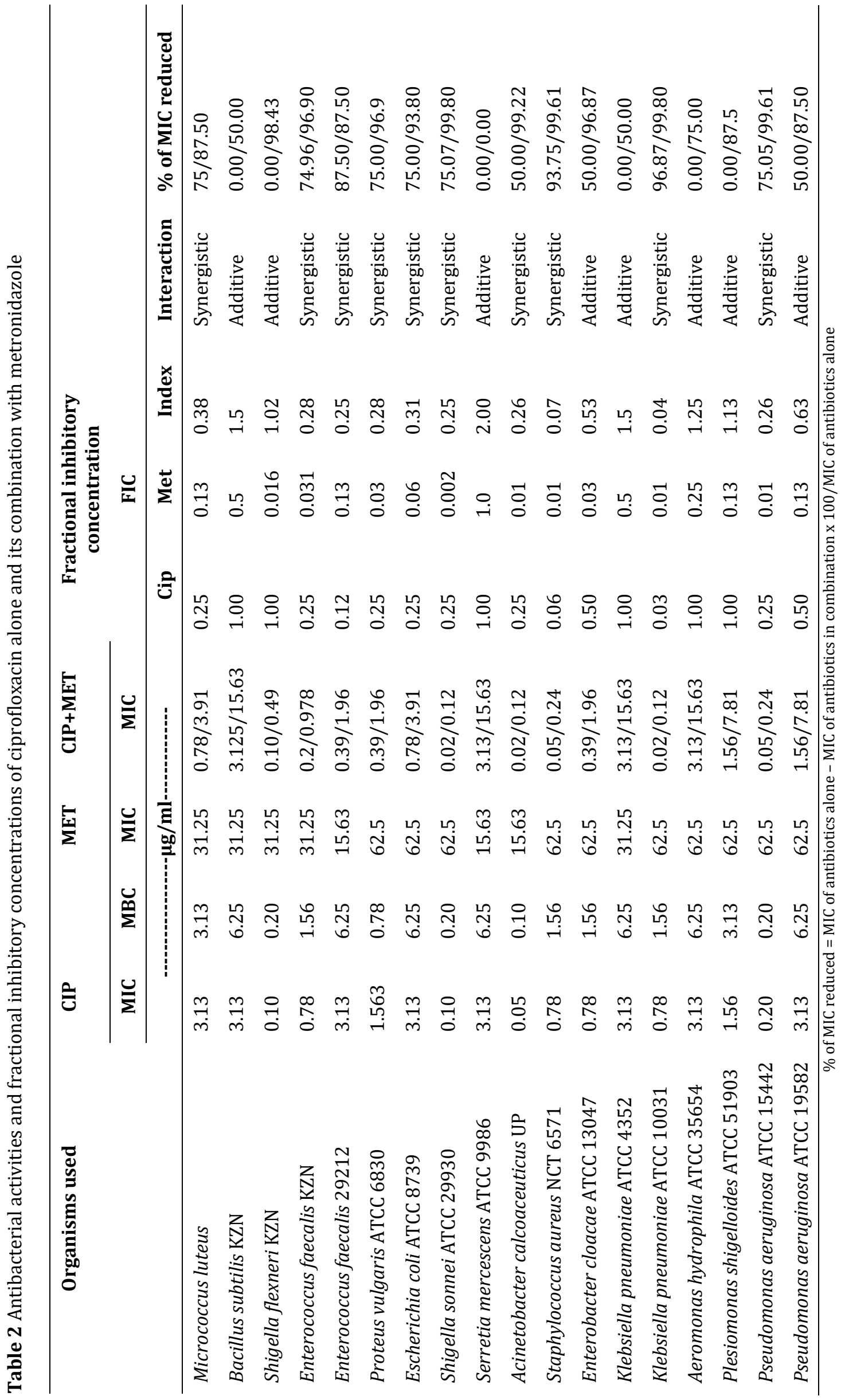


On the other hand, the MIC of ciprofloxacin ranged between 0.05 and $3.13 \mu \mathrm{g} / \mathrm{ml}$ while those of metronidazole were 15.63 and $62.5 \mu \mathrm{g} / \mathrm{ml}$. The MBC of ciprofloxacin alone ranged from 0.0978 to $6.25 \mu \mathrm{g} / \mathrm{ml}$. Acinetobacter calcaoceuticus UP, Enterococcus faecalis KZN, Shigella flexneri KZN, Staphylococcus aureus NCT 6571, Enterobacter cloacae ATCC 13047, Klebsiella pneumoniae ATCC 10031, Pseudomonas aeruginosa ATCC 15442 and Shigella sonnei ATCC 29930 were more susceptible to ciprofloxacin at concentrations less than $1 \mu \mathrm{g} / \mathrm{ml}$ while other isolates were susceptible to ciprofloxacin at concentrations ranging between 1.56 and $3.13 \mu \mathrm{g} / \mathrm{ml}$. Though the organisms were resistant to metronidazole, the MICs of the antibacterial combinations ranged between 0.02/0.12 (Cip/Met) $\mu \mathrm{g} / \mathrm{ml}$ and 3.13/1.56 (Cip/Met) $\mu \mathrm{g} / \mathrm{ml}$ against all the test bacterial isolates. Ciprofloxacin showed a significant reduction in the MICs and an increase in antibacterial activity. The percentage reduction in the MICs showed that, while the MICs of ciprofloxacin were reduced by between 0 and $96.87 \%$, those of metronidazole were reduced by between 0 and $99.80 \%$ when the two antibiotics were combined. The fractional inhibitory concentration index (FICI) showed that the interaction of ciprofloxacin and metronidazole was more of synergy (55.56 \%) than being additive/indifference (44.4\%). The FIC of ciprofloxacin ranged between 0.062 and 1.0 while those of metronidazole ranged between 0.004 and 0.125 . The FICI indicating synergistic interaction ranged between 0.035 and 0.375 and those of additive/indifference interactions ranged between 0.53 and 1.25 (Table 2).

\section{Discussion}

Drug-resistant bacteria are a critical and rapidly growing global health issue in clinical and environmental contexts [33]. It threatens human health worldwide [34] and affects treatment decisions, patient's response to treatment, and public perceptions of health care as well as increasing treatment cost, probability of treatment failures and mortality rate $[35,36]$. Drug resistance can occur through various mechanisms including enzymatic modification or degradation of a drug, pumping of a drug out of the cell by efflux pumps and modification of a drug target. While bacteria, including resistant strains, could be eliminated at high drug concentrations, drugs are co-administered for their enhanced therapeutic value $[37,38]$ to treat polymicrobial infections and infections where the etiologic agent is unknown at the start of therapy [39].

In this study, the combination of ciprofloxacin and metronidazole resulted in both synergistic and additive/indifference interactions. This study is in agreement with Alou et al. [40] and Jacqueline et al. [41] who reported that many in vitro antibiotic combinations have resulted in no interaction and/or synergistic interactions. While the synergy potentially increases efficacy, reduces toxicity, cures faster, prevents the emergence of resistance and provides broader-spectrum of activity than monotherapy regimens [42], the synergistic interactions between ciprofloxacin and metronidazole could have the potential to not only increase the rate of initial treatment response [43, 44] but could also reduce the concentration of each antibiotic needed to elicit a given effect and consequently improve the therapeutic index [45].

Although drug interactions are a concern with antibiotic use and significant drug interactions and patient harm that results from them are common concerns in clinical practice, aiming multiple targets sites in bacteria with combinations of drugs represents an important strategy for effective treatment of infection and could potentially lead to higher response rates and better clinical outcomes. By aiming at different target sites, simultaneously, in a bacterial pathogen, it is possible to obtain a more-than-additive response compared to the activity of the individual agents alone [46, 47]. The synergistic effects at the target sites will increase the efficacy of treatments, improve the selectivity and reduce offtarget effects. Hence, combining ciprofloxacin with metronidazole could have resulted in the formation of a complex compound with better antibacterial activity leading to alteration in the structural and functional features of the bacteria. Since ciprofloxacin exert its antibacterial effects by disrupting DNA synthesis to cause double stranded DNA breaks during DNA replication [48] and the activated reduced metronidazole molecule binds, inactivates and causes DNA breakage leading to DNA degradation and cell death $[49,50]$, the different modes of action of the two antibiotics may, also, be an important factor in the enhanced antibacterial efficacy observed when used in combined form. The inhibitory effects of the antibacterial combination, by increasing bacterial membrane permeability, could have also caused leakage of bacterial contents resulting cell deaths.

Since Eagle and Musselman [51] indicated that increasing dosage of drugs beyond a certain point actually decreases their effects as observed when DNA synthesis inhibitor such as ciprofloxacin [52] are used, combining ciprofloxacin with metronidazole would result in decreased dosage of the either drugs and an increased antibacterial effect without elevating single-drug concentrations to level that engender debilitating side effects in treatment of infections as Anderson et al. [53] and Dejongh et al. [54] indicated that in vitro synergy of multiple treating drugs is positively correlated with bactericidal activity and clinical outcomes. 


\section{Conclusion}

In conclusion, understanding drug interactions and its potentially beneficial effects are important. This study shows that metronidazole does not antagonize the activity of ciprofloxacin commonly used as a broad spectrum antimicrobial agent but rather, while the two antibiotics work independently, the metronidazole, also, enhance the antibacterial activity of ciprofloxacin. The combination of the two drugs may be useful in attaining better therapeutic effects in infections where polymicrobial and resistant bacterial strains may be involved.

\section{Compliance with ethical standards}

\section{Disclosure of conflict of interest}

The authors hereby declare that we do not have any conflict of interest.

\section{References}

[1] Coates A, Hu Y, Bax R and Page C. (2002). The future challenges facing the development of new antimicrobial drugs. Nature Reviews Drug Discovery, 1, 895-910.

[2] Krishnan A. (2007). Dictionary of Microbiology, (student edition), Chopsani Road, Jodhpur, 28.

[3] Esposito S and Leone S. (2007). Antimicrobial treatment for intensive care unit (ICU) infections including the role of the infectious diseases specialist. International Journal of Antimicrobial Agents, 29, 494-500.

[4] Lockhart SR, Abramson MA, Beekman SE, Gallagher G, Riedel SR, Diekma DJ, Quinn JP and Doern GV. (2007). Antimicrobial resistance among Gram-negative bacilli as causes of infections in intensive care unit patients in the United States between 1993 and 2004. Journal of Clinical Microbiology, 45, 3352-3359.

[5] Bradley JS, Guidos R, Baragona S, Bartlett JG, Rubinstein E, Zhanel GG, Tino MD, Pompliano DL, Tally F, Tipirneni P, Tillotson GS, Powers JH and Tillotson GS. (2007). Anti-infective research and development problems, challenges and solutions: the clinical practitioner perspective. Lancet Infectious Diseases, 7, 68-78.

[6] Zhanel GG, Laing NM, Nichol KA, Palatnick LP, Noreddin A, Hisanaga T, Johnson JL, the NAVRESS group and Hoban DJ. (2003). Antibiotic activity against urinary tract infection (UTI) isolates of vancomycin-resistant enterococci (VRE): results from the 2002 North America Vancomycin Resistant Enterococci Susceptibility Study (NAVRESS). Journal of Antimicrobial Chemotherapy, 52, 382-388.

[7] Raymond J and Aujard Y. (2000). Nosocomial infections in pediatric patients: a European multicenter prospective study. European Study Group. Infection Control \& Hospital Epidemiology, 21, 260-263.

[8] Schafer JJ, Goff DA, Stevenson KB and Mangino JE. (2007). Early experience with tigecycline for ventilator associated pneumonia and bacteremia caused by multidrug-resistant Acinetobacter baumannii. Pharmacotherapy, 27, 980-987.

[9] Fulton MM and Allen ER. (2005). Polypharmacy in elderly: a literature review. Journal of the American Association of Nurse Practitioners, 17(4),123-32.

[10] Hajjar ER, Cafiero AC and Hanlon JT. (2007). Polypharmacy in elderly patients. The American Journal of Geriatric Pharmacotherapy, 5(4), 345-51.

[11] Chait R, Craney A and Kishony R. (2007). Antibiotic interactions that select against resistance. Nature, 446, 668671.

[12] Hegreness M, Shoresh N, Damian D, Hartl D and Kishony R. (2008). Accelerated evolution of resistance in multidrug environments. Proceedings of the National Academy of Sciences, USA, 105, 13977-13981.

[13] Michel JB, Yeh P, Chait R, Moellering RC and Kishony R. (2008). Drug interactions modulate the potential for evolution of resistance. Proceedings of the National Academy of Sciences, USA, 105, 14918-14923.

[14] Phillips PC, Otto SP and Whitlock MC. (2000) Epistasis and the Evolutionary Process. Oxford Univ. Press; New York.

[15] Mani R, Onge RPS, Hartman JL, Giaever G and Roth FP. (2008). Defining genetic interaction. Proceedings of the National Academy of Sciences, USA, 105, 3461-3466. 
[16] Rybak MJ and McGrath BJ. (1996). Combination antimicrobial therapy for bacterial infections. Guidelines for the Clinician. Drugs, 52, 390-405.

[17] Rosholm JU, Bjerrum L, Hallas J, Worm J and Gram LF. Polypharmacy and the risk of drug-drug interactions among Danish elderly: a prescription database study. Danish Medical Bulletin, 45, 210-213.

[18] Seymour RM and Routledge PA. (1998). Important drug-drug interactions in the elderly. Drugs Aging, 12, 485494.

[19] Simonson W and Feinberg JL. (2005). Medication-related problems in the elderly. Defining the issues and identifying solutions. Drugs Aging, 22(7), 559-69.

[20] Kuijpers MA, van Marum RJ, Egberts AC and Jansen PA. (2008). Relationship between polypharmacy and underprescribing. British Journal of Clinical Pharmacology, 65(1), 130-3.

[21] Milton JC, Hill-Smith I and Jackson SHD. (2008). Prescribing for older people. BMJ, 336(7644), 606-9.

[22] Boyle N, Naganathan V and Cumming RG. (2010). Medication and falls: risk and optimization. Clin Geriatr Med, 26(4), 583-605.

[23] Olajuyigbe 00, Oyedeji 0 and Adedayo 0. (2014). Evaluation of the in vitro interaction of amoxicillin and cotrimoxazole antibiotics against resistant bacterial strains. Journal of Applied Pharmaceutical Science, 4(1), 94100 .

[24] Guzey C and Spigset 0. (2002). Genotyping of drug targets: a method to predict adverse drug reactions? Drug Safety, 25, 553-560.

[25] Roberts RL, Begg EJ, Joyce PR and Kennedy MA. (2002). How the pharmacogenetics of cytochrome P450 enzymes may affect prescribing. New Zealand Medical Journal, 115, 137-140.

[26] Richard S, Lynn SM and Avery CG. (2007). Antimicrobial susceptibility testing protocols. New York: CRC Press.

[27] Cheesbrough M. (2002). Medical Laboratory Manual for Tropical Countries, ELBS ed; Tropical health technology publications and Butterworth-Heinemann Ltd: Cambridge, UK, 2, 2-392.

[28] Clinical and Laboratory Standard Institute (CLSI). (2008). Performance standards for Antimicrobial Susceptibility Testing Eighteenth informational supplement. M100-S18 2008, 28(1), 46-52.

[29] Kelly DJ. (1992). Serum concentrations of penicillin, doxycycline, and ciprofloxacin during prolonged therapy in rhesus monkeys. Journal of infectious Diseases, 166, 1184-7.

[30] Cheesbrough M. (2006). District Laboratory Practice in Tropical Countries. $1^{\text {st }}$ Edn., Cambridge University Press, Cambridge, UK., ISBN-10: 0521665450, 434.

[31] Petersen PJ, Labthavikul P, Jones CH and Bradford PA. (2006). In vitro antibacterial activities of tigecycline in combination with other antimicrobial agents determined by chequerboard and time-kill kinetic analysis. Journal of Antimicrobial Chemotherapy, 57, 573-576.

[32] Mandal S, Mandal MD and Pal NK. (2004). Evaluation of combination effect of ciprofloxacin and cefazolin against Salmonella enteric serovar typhi isolates by in vitro methods. Calicut Medical Journal, 2(2), e2.

[33] Wright GD, (2010). Antibiotic resistance in the environment: a link to the clinic? Microbiology, 13, 589-594.

[34] Hogberg LD, Heddini A and Cars 0. (2010). The global need for effective antibiotics: challenges and recent advances. Trends in Pharmacological Sciences, 31, 509-515.

[35] Sosa AJ, Byarugaba DK, Amábile-Cuevas CF, Hsueh P.R, Kariuki S and Okeke IN (eds). (2010). Antimicrobial Resistance in Developing Countries. New York: Springer.

[36] Eisenberg JNS, Goldstick J, Cevallos W, Trueba G, Levy K, Scott J, Percha B, Segovia R, Ponce K, Hubbard A, Marrs C. (2011). In-roads to the spread of antibiotic resistance: regional patterns of microbial transmission in northern coastal Ecuador. Journal of the Royal Society Interface, 9(70), 1029-1039.

[37] Lehár J, Krueger AS, Avery W, Heilbut AM, Johansen LM, Price ER, Rickles RJ, Short Iii GF, Staunton JE, Jin X, Lee MS. (2009). Synergistic drug combinations tend to improve therapeutically relevant selectivity. Nature biotechnology, 27(7), 659-666.

[38] Farha MA and Brown ED. (2010). Chemical probes of Escherichia coli uncovered through chemical-chemical interaction profiling with compounds of known biological activity. Chemistry \& Biology, 17, 852-862. 
[39] Gorbach SL. (1994). Piperacillin/tazobactam in the treatment of polymicrobial infections. Intensive Care Medicine, 20 Suppl 3, S27-34.

[40] Alou L, Cafini F, Sevillano D, Unzueta I and Prieto J. (2004). In vitro activity of mupirocin and amoxicillinclavulanate alone and in combination against staphylococci including those resistant to methicillin. International Journal of Antimicrobial Agents, 23, 513-6.

[41] Jacqueline C, Navas D, Batard E et al.. (2005). In vitro and in vivo synergistic activities of linezolid combined with subinhibitory concentrations of imipenem against methicillin resistant Staphylococcus aureus. Antimicrobial Agents Chemotherapy, 49, 45-51.

[42] Marr KA, Boeckh M, Carter RA, Kim HW and Corey L. (2004). Combination antifungal therapy for invasive aspergillosis. Clinical Infectious Diseases, 39, 797-802.

[43] Azmi AS, Wang Z, Philip PA, Mohammad RM and Sarkar FH. (2010). Proof of Concept: Network and Systems Biology Approaches Aid in the Discovery of Potent Anticancer Drug Combinations. Molecular Cancer Therapeutics, 9, 3137-3144.

[44] Glickman MS and Sawyers CL. (2012). Converting cancer therapies into cures: Lessons from infectious diseases. Cell, 148, 1089-1098.

[45] Olajuyigbe 00, Coopoosamy RM and Afolayan AJ. (2017). Effects and time-kill assessment of Amoxicillin in combination with Chloramphenicol against bacteria of clinical importance. Acta Biochimica Polonica, 64(11), 15 .

[46] Fitzgerald JB, Schoeberl B, Nielsen UB and Sorger PK. (2006). System biology and combination therapy in the quest for clinical efficacy. Nature Chemical Biology, 2, 458-466.

[47] Lehár J, Stockwell BR, Giaever G and Nislow C. (2008). Combination chemical genetics. Nature Chemical Biology, 4, 674-681.

[48] Petri WAJ. (2005). Antimicrobial agents: sulphonamides, trimethoprim-sulfmethaxozole, quinolones, and agents for urinary tract infections. Goodman and Gilman's The Pharmocol basis of Ther, 10, 1111-1126.

[49] Diniz CG, Santos SG, Pestana AC, Farias LM, Carvalho MA, Auxiliadora M. (2000). Chromosomal breakage in the Bacillus fragilis group induced by metronidazole treatment. Anaerobe, 6, 149-53.

[50] Chen YH, Ko WC and Hsueh PR. (2013). Emerging resistance problems and future perspective in pharmacotherapy. Expert Opinion on Pharmacotherapy, 14, 587-596.

[51] Eagle $\mathrm{H}$ and Musselman AD. (1948). The rate of bactericidal action of penicillin in vitro as a function of its concentration and its paradoxically reduced activity at high concentrations against certain organisms. The Journal of Experimental Medicine, 88, 99-131

[52] Lewin CS, Morrissey I and Smith JT. (1991). The mode of action of quinolones: the paradox inactivity of low and high concentrations and activity in the anaerobic environment. European Journal of Clinical Microbiology \& Infectious Diseases, 10, 240-248.

[53] Anderson ET, Young LS and Hewitt WL. (1978). Antimicrobial synergism in therapy of Gram Negative Rod Bacteremia. Chemotherapy, 24(1), 45-54.

[54] Dejongh CA, Joshi JH, Newman KA, Moody MR, Wharton R, et al. (1986). Antibiotic Synergism and Response in Gram-Negative Bacteremia in Granulocytopenic Cancer - Patients. The American Journal of Medicine, 80, 96-100.

\section{How to cite this article}

Adeoye-Isijola MO, Osopale BA and Olajuyigbe 00. (2019). In vitro influence of metronidazole on the activities of ciprofloxacin against clinically important bacterial isolates. GSC Biological and Pharmaceutical Sciences, 6(3), 31-39. 